DETC2000/MECH-14119

\title{
Optimization-Based Design of a Fully-Compliant Bistable Micromechanism
}

\author{
Matthew B. Parkinson Brian D. Jensen \\ Department of Mechanical Engineering and Applied Mechanics \\ University of Michigan \\ Ann Arbor, Ml \\ Gregory M. Roach \\ Department of Mechanical Engineering \\ Brigham Young University \\ Provo, UT
}

\begin{abstract}
Micro-bistable mechanisms are used in microswitches and microvalves to reduce power consumption as power is applied only to switch states. Many of the bistable mechanism designs that have been presented incorporate rigid-body joints. These joints introduce unwanted friction and poor repeatability into the mechanism motion. A fully-compliant mechanism avoids these problems. Optimization techniques were used to find fully-compliant bistable micromechanism designs. The chosen objective was to minimize the displacement required between the two stable positions. Two families of designs were considered: those where the actuator was integral to the device and those where it remained in contact only during actuation. Mechanism designs are presented and are currently in the process of fabrication.
\end{abstract}

\section{INTRODUCTION}

Mechanically bistable micromechanisms maintain either of two stable positions without the need of constant power input. This is attractive for systems with power constraints because power is supplied only to switch the mechanisms between states. For example, several examples of bistable mechanisms for micro-relays or micro-switches have been presented (Hälg, 1990; Matoba et al., 1994; Kruglick and Pister, 1998; Sun et al., 1998, Jensen et al., 1999). Bistable microvalves have also been discussed which remain either open or closed (Wagner et al., 1996; Goll et al., 1996; Shinozawa et al., 1997; Schomburg and Goll, 1998). A bistable fiber-optic switch (Hoffman et al., 1998) and a bistable system to produce a force for assembling microparts (Vangbo and Bäcklund, 1998) have also been suggested.

These bistable micromechanisms generally fall into one of two broad categories. Many of the devices presented use residual stress in deposited films to create beam buckling, a well-known bistable phenomenon. Other devices use strain energy storage and a mixture of rigid-body and compliant joints to create bistable mechanisms. This approach is especially useful in standard MEMS fabrication processes, such as Cronos' MUMPs or Sandia National Laboratories' SUMMiT. The goal of stress-free polysilicon inherent in these processes makes small-size buckling designs infeasible. Unfortunately, however, friction in rigid-body joints creates a considerable increase in power requirements, as well as a corresponding reduction in reliability due to stiction. Moreover, bistable micromechanisms which include rigid-body joints suffer from poor repeatability. The large clearances typical of these joints induce variation in the locations at which the mechanism is stable (Jensen et al., 1999). Hence, a more robust design would consist of one flexible piece of material which would not rely on residual stress for its bistable behavior. Such a design would realize the advantages of compliant mechanisms, such as friction-free operation, no backlash, and no wear (Ananthasuresh and Kota, 1996).

Several design constraints peculiar to MEMS impose added complexity to the design problem. For example, stress in thin flexural pivots tends to prevent large motions, requiring that the mechanism design involve only small angular motions over its range of deflection. In addition, during mechanism motion, some of the energy stored in the mechanism would have to work 

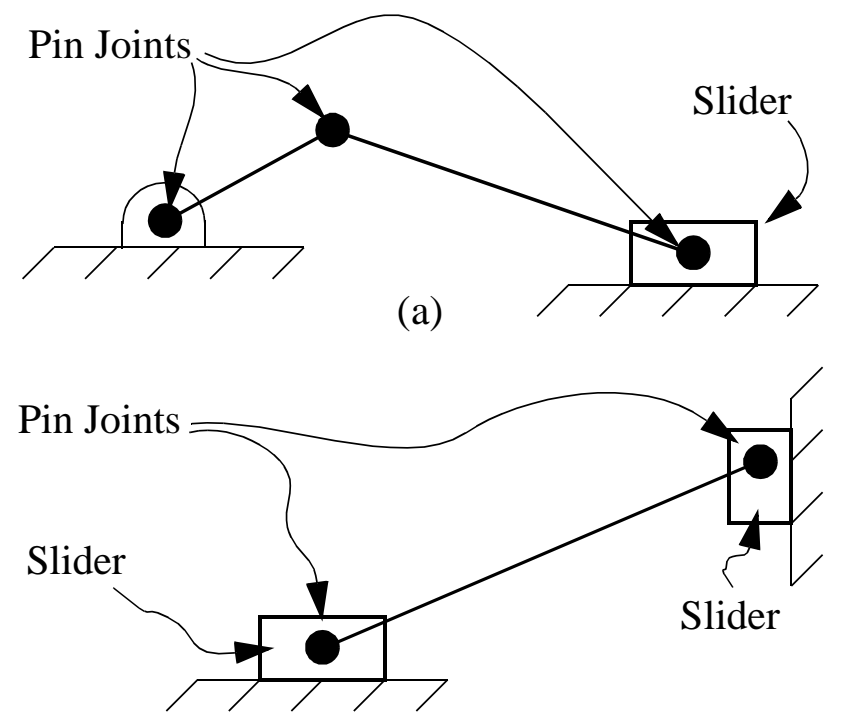

(b)

Figure 1. The slider-crank (a) and the doubleslider (b) mechanism types

to force the mechanism into a second stable position, while some of the stored energy would necessarily work to return to the initial fabrication position. In order to better explore the design space, we decided to use optimization techniques, which would allow us to evaluate many designs very quickly, examining feasible designs in further depth while discarding infeasible designs.

\section{MECHANISM TYPOLOGY}

The first step in the mechanism design was to choose a basic topology for the device. For simplicity in attaching the device to an actuator, linear motion was desired for the input. A kinematic slider was therefore chosen as the input link. With this basic stipulation, we considered two basic mechanism typologies, the slider-crank and the double-slider mechanisms, shown in Figure 1. Each type contains the required slider joint, and prior work had shown both to be excellent candidates for bistable mechanisms (Jensen, 1998).

A preliminary analysis was performed for each mechanism type to determine its suitability for fully-compliant bistable mechanism design. For this analysis, each mechanism type was modeled as a fully-compliant mechanism. The details of the modeling for the double-slider type are given below, and the modeling of the slider-crank type is similar. Preliminary optimization performed on each model showed that while the double-slider design space contained feasible bistable designs, the slider-crank design space contained few, if any, feasible bistable designs. Thus, the double-slider mechanism type was chosen for further study.

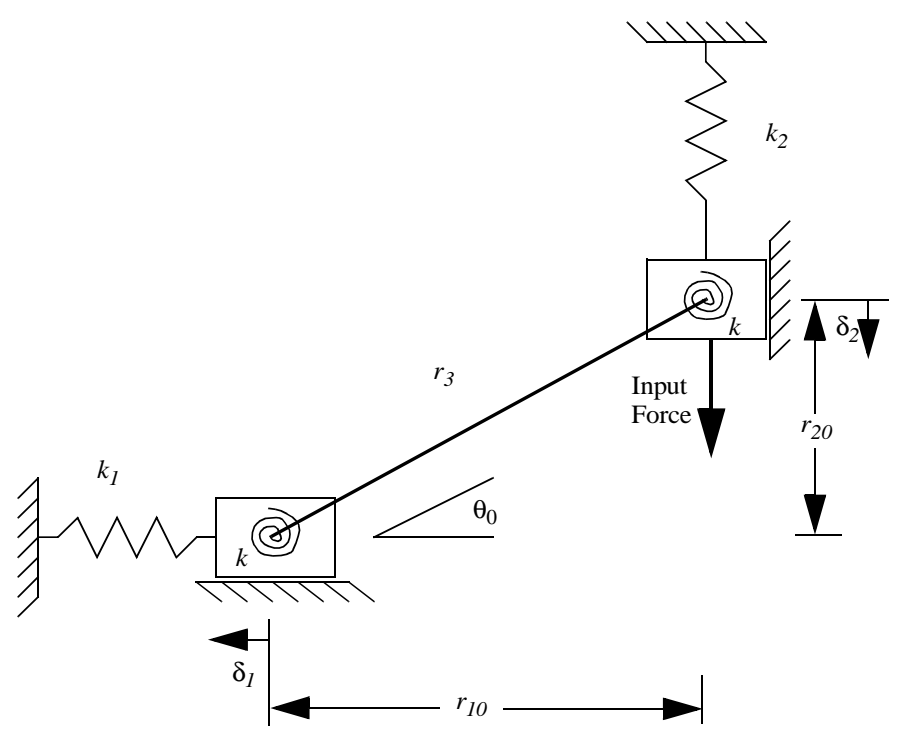

Figure 2. The kinematic model of the bistable mechanism design

\section{MODEL DEVELOPMENT}

To make the mechanism fully compliant, thin flexible segments could be used in place of the pin joints, and any of several common linear suspensions, such as the folded beam suspension, could be used in place of the sliders.

A simple kinematic model of a fully-compliant doubleslider mechanism requires torsional or translational springs to be placed at each mechanism joint, as shown in Figure 2. This mechanism model is based on pseudo-rigid-body modeling, in which thin flexible segments (known as small-length flexural pivots) are modeled as pin joints, with torsional springs to represent bending stiffness (Howell and Midha, 1994). For simplicity, we require that the two small-length flexural pivots have the same geometry and consequently the same stiffness $k$. The mathematical model of the mechanism may then be formulated.

First, the dependent design variables must be calculated from the independent variables:

$$
k=\frac{t w^{3} E}{12 l}
$$

where $t$ is the out-of-plane thickness of the flexural pivots, $w$ is the in-plane width of each pivot, $E$ is the elastic modulus of the material, and $l$ is the length of each pivot. Also,

$$
r_{3}=\frac{r_{10}}{\cos \left(\theta_{0}\right)}
$$




$$
r_{20}=r_{10} \tan \left(\theta_{0}\right)
$$

Then, for each deflection $\delta_{2, i}$, where $i=1 \ldots n$ and $n$ is the number of loadsteps, calculate the intermediate and final positions:

$$
\begin{gathered}
r_{2, i}=r_{20}-\delta_{2, i} \\
\theta_{i}=\operatorname{asin}\left(r_{2, i} / r_{3}\right) \\
\delta_{1, i}=r_{3} \cos \left(\theta_{i}\right)-r_{10}
\end{gathered}
$$

and calculate the deflection of the angles

$$
\begin{gathered}
\psi_{1, i}=\theta_{0}-\theta_{i} \\
\psi_{2, i}=-\psi_{1, i}
\end{gathered}
$$

Finally, calculate the forces in the deflected position

$$
\begin{gathered}
g_{1, i}=\frac{1}{\sqrt{r_{3}^{2}-r_{2, i}^{2}}} \\
g_{2, i}=-r_{3} \sin \left(\theta_{i}\right) g_{1, i} \\
F_{i}=g_{1, i} k\left(\psi_{2, i}-\psi_{1, i}\right)+g_{2, i} k_{1} \delta_{1, i}-k_{2} \delta_{2, i}
\end{gathered}
$$

where $g_{1, i}$ and $g_{2, i}$ are the kinematic coefficients. Finally, calculate stresses in the small-length flexural pivots

$$
\sigma_{j, i}=\frac{w \psi_{j, i} E}{2 l} \text { where } j=1,2
$$

The kinematic model shown in Figure 2 may be converted to the device schematic shown in Figure 3. The vertical slider is approximated using the symmetry of the device, as illustrated in Figure 3a. The horizontal slider and its associated spring are formed using a fixed-fixed beam, as shown in Figure $3 b$. Additionally, the pin joints and torsional springs are replaced by small-length flexural pivots.

The mathematical model presented above is useful for optimization studies, where it may be quickly evaluated for a large number of candidate designs. It is, however, a less accurate approximation than a nonlinear finite element model of the device. Therefore, we also developed a finite element model of the device using beam elements. We compared the mathematical model to the finite element model for a few random designs, and found that it nearly approximated the force and stress results. Consequently, the mathematical model was used for optimization analysis, and the computationally intensive finite element model was reserved for verifying the feasibility of the resulting design.

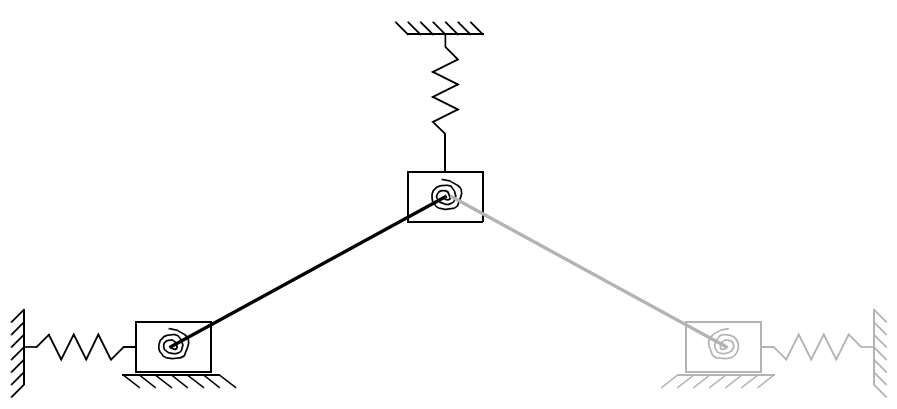

(a)

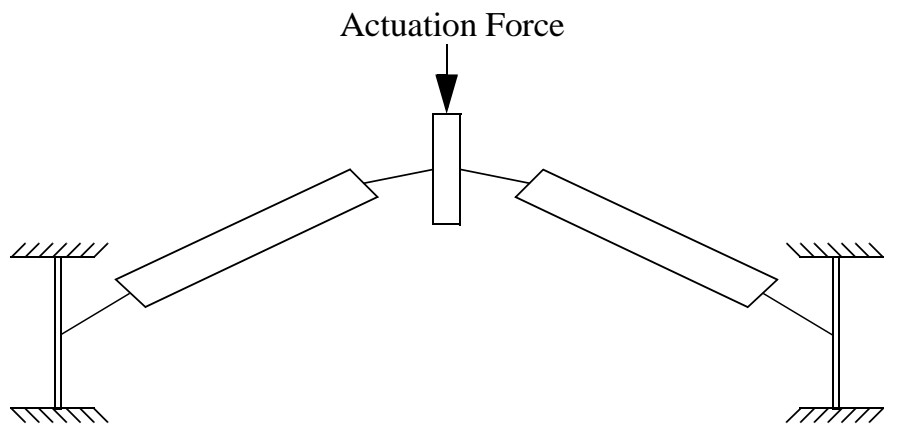

(b)

Figure 3. (a) Schematic showing symmetry of the half-model from Figure 2. (b) Layout schematic for the fully-compliant bistable mechanism

\section{THE OPTIMIZATION MODEL}

In evaluating the performance of a mechanism, the material and manufacturing process are taken into account through the selection of $E, t$, and $w$. This work was done with the SUMMiT process in mind, and the parameter values (Table 1) reflect that. Designs for MUMPs were also generated, but for brevity they are not presented here.

The geometry of the fixed-fixed beam and the type of actuation are reflected in the selection of $k_{1}$ and $k_{2}$, respectively. The spring stiffness of the top spring, $k_{2}$, is chosen to reflect two

Table 1: Parameter values for the optimization problem

\begin{tabular}{|c|c|c|c|}
\hline Symbol & Description & Units & Value \\
\hline$w$ & $\begin{array}{c}\text { width of small-length } \\
\text { flexural pivots }\end{array}$ & $\mu \mathrm{m}$ & 1.5 \\
\hline$t$ & out-of-plane thickness & $\mu \mathrm{m}$ & 2 \\
\hline$E$ & modulus & $\mathrm{GPa}$ & 165 \\
\hline$k_{1}$ & side spring & $\mathrm{N} / \mathrm{m}$ & $75-2500$ \\
\hline$k_{2}$ & top spring & $\mathrm{N} / \mathrm{m}$ & $0.0-0.1$ \\
\hline
\end{tabular}




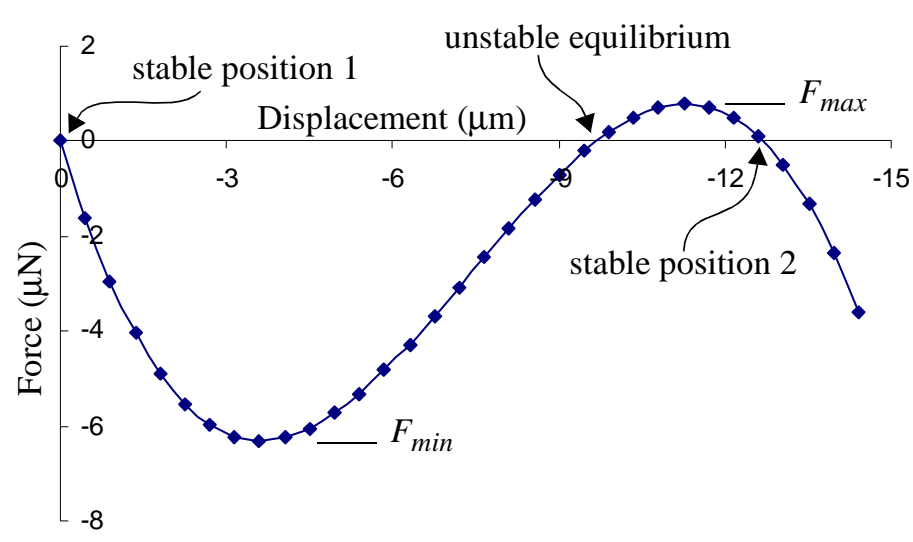

Figure 4. A force / displacement curve for a bistable mechanism.

different types of actuators that could be used with the mechanism. An electrostatic (comb) actuator would typically be rigidly connected to the device to allow motion in both directions. Thus, due to symmetry, $k_{2}$ would be equal to half of the spring constant of the comb drive suspension. A reasonable value for the SUMMiT process is $0.1 \mathrm{~N} / \mathrm{m}$. On the other hand, a thermal actuator would not be rigidly connected to the device, so that no spring force would act to oppose the motion of the slider. Thus, in this case, $k_{2}=0$. The side spring, $k_{1}$, can take on a number of different values, depending on the chosen stiffness of the beam which acts as the spring. The remaining variables are the design variables, $\boldsymbol{x}=\left[\theta_{0}, r_{10}, l\right]$, as shown in Figure 2 .

We first determine the characteristics of a feasible mechanism. As mentioned previously, the reaction force and stress in the members are calculated at each of the loadsteps as the mechanism is deflected. These values are stored so that $F_{\text {max }}, F_{\text {min }}$, and $\sigma_{\text {max }}$ for the entire deflection may be obtained. The maximum stress incurred must remain below the allowable stress in polysilicon. At the same time, the force $\left(F_{\max }\right)$ needs to change sign at some point throughout its travel (indicating that the mechanism is bistable). Moreover, $F_{\max }$ must exceed a minimum force $\left(F_{\text {bistable }}\right)$ to remain in the second stable position in the presence of random vibrations. A plot of a design meeting the force criteria is shown in Figure 4.

A third constraint is imposed to maintain the quality of the modeling approximation. The ratio of the small-length flexural pivots to the length of the leg to which they are attached needs to be small enough (less than a parameter $\lambda$ ) that the pseudorigid-body model (PRBM) is accurate. Finally, subject to these constraints, the distance through which the mechanism must be actuated needs to be as small as possible-both to reduce the overall size of the mechanism and to allow the use of thermal actuators. The magnitude of $F_{\min }$, the chosen value for $k_{2}$, and the resulting minimum $r_{20}$ determine the type of actuator that can be used.
The optimization problem is then

$$
\underset{\boldsymbol{x}}{\operatorname{minimize}} r_{20}
$$

subject to

$$
\begin{gathered}
\max \left(\sigma_{\text {max }, i}\right) \leq \sigma_{Y} \\
\max \left(F_{\text {max }, i}\right) \geq F_{\text {bistable }} \\
\frac{l}{r_{3}} \leq \lambda
\end{gathered}
$$

where $i=1 \ldots n$

The problem was solved using the Generalized Reduced Gradient (GRG) method. Information on the particular algorithm used is found in Parkinson and Wilson (1988). To verify that the solutions were global optima, the stochastic algorithm, simulated annealing (SA), was used. Specific discussions of the algorithm may be found in Kirkpatrick, et. al. (1983). For this problem, simulated annealing was run for 10,000 cycles. The resulting answer was very near to that obtained using GRG. Quick use of the GRG method, using the SA answer as a starting point, yielded the original answer. A monotonicity analysis was not performed due to the nonimplicit nature of the problem formulation.

This design was then analyzed using the finite element model to verify that the mechanism was indeed bistable and that the other constraints were still satisfied. We found that while the nature of the mechanism was the same (it was bistable), the PRBM usually slightly underapproximated both $\sigma_{\max }$ and $F_{\max }$. To compensate for this, a safety factor was incorporated into the constraints on each of these values. The final numerical optimization model was then

$$
\begin{array}{cc}
\underset{\boldsymbol{x}}{\operatorname{minimize}} & r_{20} \\
\text { subject to } & \max \left(\sigma_{\max , i}\right) \leq 800 \mathrm{MPa} \\
& \max \left(F_{\max , i}\right) \geq 2 \mu \mathrm{N} \\
\frac{l}{r_{3}} \leq 0.1
\end{array}
$$

Using this model, the procedure outlined above (running GRG, then verifying global solution using SA) was repeated for two families of designs: those where the actuator was not attached to the device $\left(k_{2}=0\right)$ and those where it was $\left(k_{2}=0.1\right)$. Depending on the dimensions of the fixed-fixed beam $k_{1}$ varies from $75 \mathrm{~N} / \mathrm{m}$ to $2500 \mathrm{~N} / \mathrm{m}$. For each of the design families, a parametric study of the effect of $k_{l}$ across this range was performed. The results are plotted in Figure 5.

As Figure 5 shows, the design family in which the actuator lets go of the mechanism required less displacement to achieve its second stable position than those in which the actuator remained attached to the mechanism. This was anticipated since more strain energy is needed to overcome the resistance of the attached actuator. The parameter $k_{l}$ has the same effect on both 


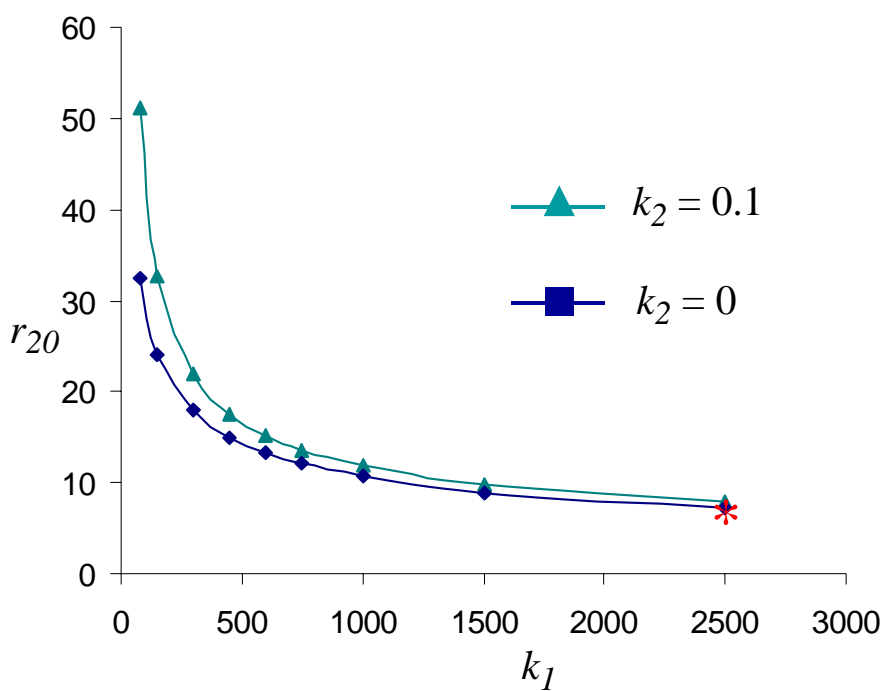

Figure 5. Curves for the two design families showing the results of a parametric study in $k_{1}$. The * denotes the design detailed in the paper body.

families: as it increases, the displacement required to achieve bistability decreases. The use of a thermal actuator generally requires small displacement, $r_{20}$, so the designs with larger $k_{l}$ would be favored. If greater displacement were desired, an electrostatic actuator could be used with the designs for the smaller $k_{1}$.

\section{A PARTICULAR DESIGN}

The parameter and variable values describing one of the resulting designs (denoted by $*$ in Figure 5 ) are given in Table 2 below. Note that the maximum force $\left(F_{\max }\right)$ is below the stated constraint value from Eq. (14). As discussed earlier, the $2 \mu \mathrm{N}$ value for the optimization model incorporates a safety factor because this model tends to overpredict $F_{\max }$. This ensures that the correct value of $F_{\max }$, calculated from the finite element model, is still adequate. A scale layout of this sample design is shown in Figure 6. The force-deflection curve in Figure 4 was also calculated for this design. In the layout, leads have been placed at the second stable position to allow the device to function as a micro-relay. The deflection required before snapthrough is about $9.5 \mu \mathrm{m}$, which is the smallest deflection in the family of designs generated in this paper. This deflection is well within the range of demonstrated thermal actuators (Cragun and Howell, 1999). This and other designs from the generated design families are in the process of being fabricated.

A contour plot of the design space surrounding the optimum is shown in Figure 7. The contours are the objective function. For this plot $q_{0}$ is held fixed while $r_{10}$ and $l$ are varied. Note that the design is near the $\sigma_{\max }$ boundary, but that only the $l / r_{3}$ and $F_{\max }$ constraints are active, bounding the optimum from above and below, respectively. The type of constraint activity

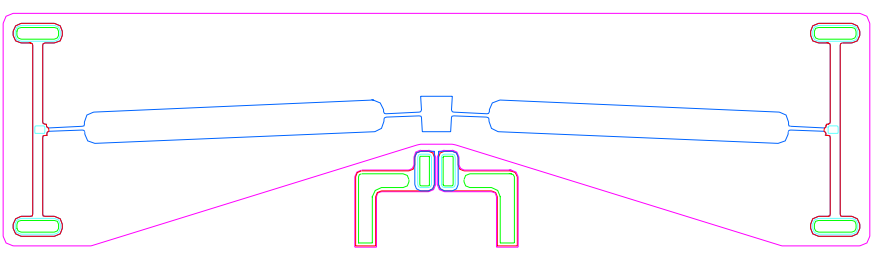

Figure 6. Layout for a bistable switch showing location of contact pads for the switch to be closed in the second stable position

shown in Figure 7 is typical of designs with high values of $k_{l}$ and $k_{2}=0$. As $k_{1}$ increases and for all the designs where $k_{2}=$ 0.1 , all three constraints are active.

Note also that the $l / r_{3}$ constraint is a modeling constraint imposed to keep the designs in the region where the PRBM is sufficiently accurate. Its activity indicates that while the designs above are good bistable designs, they are not truly optimal. More accurate optimization models would thus allow improvement in the designs.

Table 2: Variable and function values describing a bistable micromechanism

\begin{tabular}{|c|c|c|c|}
\hline Symbol & Description & Units & Value \\
\hline$w$ & $\begin{array}{l}\text { width of small-length } \\
\text { flexural pivots }\end{array}$ & $\mu \mathrm{m}$ & 1.5 \\
\hline$t$ & $\begin{array}{l}\text { out-of-plane thick- } \\
\text { ness }\end{array}$ & $\mu \mathrm{m}$ & 2 \\
\hline$E$ & modulus & $\mathrm{GPa}$ & 165 \\
\hline$k_{1}$ & side spring & $\mathrm{N} / \mathrm{m}$ & 2500 \\
\hline$k_{2}$ & top spring & $\mathrm{N} / \mathrm{m}$ & 0.0 \\
\hline$\theta_{0}$ & initial angle & radians & 0.042 \\
\hline$r_{10}$ & $\begin{array}{l}\text { mechanism horizon- } \\
\text { tal half-length }\end{array}$ & $\mu \mathrm{m}$ & 171 \\
\hline I & $\begin{array}{l}\text { length of small-length } \\
\text { flexural pivots }\end{array}$ & $\mu \mathrm{m}$ & 17.1 \\
\hline $\mathrm{s}_{\max }$ & maximum stress & $\mathrm{MPa}$ & 620 \\
\hline$F_{\max }$ & maximum force & $\mu \mathrm{N}$ & 0.79 \\
\hline$F_{\min }$ & minimum force & $\mu \mathrm{N}$ & -6.31 \\
\hline$r_{20}$ & objective function & $\mu \mathrm{m}$ & 7.20 \\
\hline
\end{tabular}




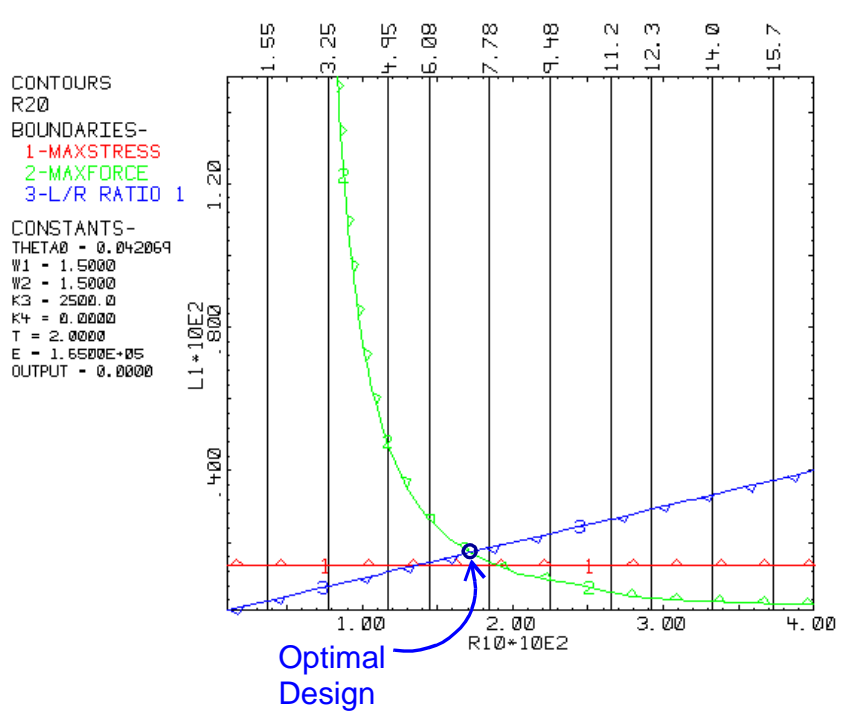

Figure 7. A contour plot of the design space surrounding an optimal design.

\section{CONCLUSION}

In this paper, optimization techniques were used to find fully-compliant bistable micromechanism designs based on the pseudo-rigid-body model. These techniques were used to make an efficient search of the design space. Once feasible designs were found, they were optimized to minimize the displacement required between the two stable positions. Two families of designs were considered: those where the actuator remained attached to the device and those where it did not. Mechanism designs for both SUMMiT and MUMPs are currently in the process of fabrication.

Because the mechanism contains no rigid-body joints, its operation is friction-free, with no backlash or wear. Stiction can be largely avoided using appropriate non-stick coatings (de Boer and Michalske, 1999). These advantages lead to smaller power consumption, more accurate motion, and greater reliability. Moreover, the device does not rely on residual stress for its bistable action, allowing it to be used in standard MEMS prototyping processes. These properties make the mechanism desirable for such applications as microswitching.

Further improvements can be made. Recognizing the inaccuracies and limitations of the PRBM when applied to this problem, the optimization routine could be run on the more time-consuming finite element model. We anticipate that the removal of the constraints placed on the small-length flexural pivots (necessary when using the PRBM) will allow the generation of designs with even smaller values for $r_{20}$. A new problem formulation involving the reduction in size of the entire mechanism could also be utilized. These improvements are currently being researched.

\section{ACKNOWLEDGEMENTS}

We would like to thank the Department of Mechanical Engineering and Applied Mechanics at the University of Michigan and the Department of Mechanical Engineering at Brigham Young University for the use of computing resources used in this work. This work is supported under a National Science Foundation Graduate Research Fellowship.

\section{REFERENCES}

Ananthasuresh, G.K. and Kota, S., 1996, "The Role of Compliance in the Design of MEMS," Proceedings of the 1996 ASME Design Engineering Technical Conferences, 96-DETC/ MECH-1309.

Cragun, R., and Howell, L.L., 1999, "Linear Thermomechanical Microactuators," Microelectromechanical Systems (MEMS), 1999 ASME International Mechanical Engineering Congress and Exposition, pp. 181-188.

de Boer, M.P., and Michalske, T.A., 1999, “Accurate Method For Determining Adhesion of Cantilever Beams," Journal of Applied Physics, Volume 86, pp. 817-827.

Goll, C., Bacher, W., Buestgens, B., Maas, D., Menz, W., and Schomburg, W.K., 1996, "Microvalves with Bistable Buckled Polymer Diaphragms," Journal of Micromechanics and Microengineering, Vol. 6, No. 1, pp. 77-79.

Hälg, B., 1990, "On A Nonvolatile Memory Cell Based on Micro-electro-mechanics," IEEE Micro Electro Mechanical Systems 1990, pp. 172-176.

Hoffman, M., Kopka, P., and Voges, E., 1998, "Bistable Micromechanical Fiber-Optic Switches on Silicon," Proceedings of the 1998 IEEE/LEOS Summer Topical Meeting, pp. 31-32.

Howell, L.L. and Midha, A., 1994, "A Method for the Design of Compliant Mechanisms with Small-Length Flexural Pivots," ASME Journal of Mechanical Design, Vol. 116, No. 1, pp. 280290.

Jensen, B.D., Howell, L.L., and Salmon, L.G., 1999, "Design of Two-Link, In-Plane, Bistable Compliant Micro-Mechanisms," ASME Journal of Mechanical Design, Vol. 121, No. 3, pp. 416423.

Jensen, Brian D., 1998, Identification of Macro- and MicroCompliant Mechanism Configurations Resulting in Bistable Behavior, M.S. Thesis, Brigham Young University, Provo, UT.

Kirkpatrick, S., Gelatt, C.D., and Vecchi, M.P., 1983, "Optimization by Simulated Annealing," Science, vol. 22, no. 459 , p. 671.

Kruglick, E.J.J. and Pister, K.S.J., 1998, "Bistable MEMS Relays and Contact Characterization," 1998 Solid-State Sensor and Actuator Workshop, pp. 333-337. 
Matoba, H., Ishikawa, T., Kim, C., and Muller, R.S., 1994, “A Bistable Snapping Mechanism," IEEE Micro Electro

Mechanical Systems 1994, pp. 45-50.

Parkinson, A.R., and Wilson, M., 1988, "Development of Hybrid GRG-SQP Algorithm for Constrained Nonlinear Programming," J of Mech. Trans. and Automation in Design, Trans. ASME, vol. 110, p. 308.

Schomburg, W.K. and Goll, C., 1998, "Design Optimization of Bistable Microdiaphragm Valves," Sensors and Actuators, A: Physical, Vol. 64, No. 3, pp. 259-264.

Shinozawa, Y., Abe, T., and Kondo, T., 1997, "Proportional Microvalve Using a Bi-Stable Magnetic Actuator," Proceedings of the 1997 IEEE 10th Annual International Workshop on Micro
Electro Mechanical Systems (MEMS), pp. 233-237.

Sun, X., Farmer, K.R., and Carr, W., 1998, "Bistable Microrelay Based on Two-Segment Multimorph Cantilever Actuators", Proceedings of the 1998 IEEE 11th Annual International Workshop on Micro Electro Mechanical Systems (MEMS), pp. 154-159.

Vangbo, M, and Bäcklund, Y., 1998, “A Lateral Symmetrically Bistable Buckled Beam,” Journal of Micromechanics and Microengineering, Vol. 8, pp. 29-32.

Wagner, B., Quenzer, H.J., Hoershelmann, S., Lisec, T., and Juerss, M., 1996, "Bistable Microvalve with Pneumatically Coupled Membranes," Proceedings of IEEE Micro Electro Mechanical Systems 1996, p. 384-388. 